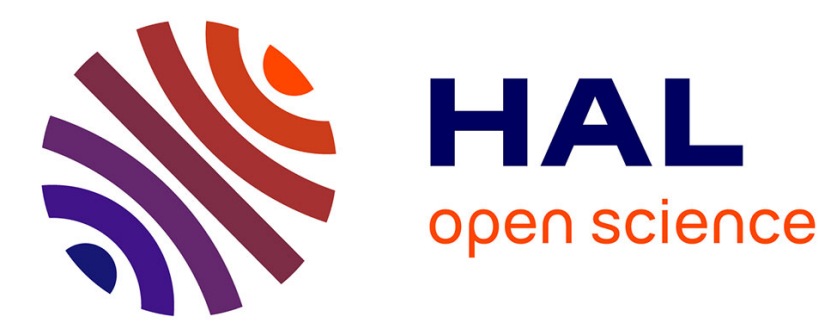

\title{
Genetic algorithm coupled with a deterministic method for optimization in electromagnetics
}

Joao Antonio Vasconcelos, R. R. Saldanha, Laurent Krähenbühl, Alain Nicolas

\section{To cite this version:}

Joao Antonio Vasconcelos, R. R. Saldanha, Laurent Krähenbühl, Alain Nicolas. Genetic algorithm coupled with a deterministic method for optimization in electromagnetics. IEEE Transactions on Magnetics, 1997, 33 (2), part 2, 1860 - 1863. 10.1109/20.582645 . hal-00359821

\section{HAL Id: hal-00359821 \\ https://hal.science/hal-00359821}

Submitted on 9 Feb 2009

HAL is a multi-disciplinary open access archive for the deposit and dissemination of scientific research documents, whether they are published or not. The documents may come from teaching and research institutions in France or abroad, or from public or private research centers.
L'archive ouverte pluridisciplinaire HAL, est destinée au dépôt et à la diffusion de documents scientifiques de niveau recherche, publiés ou non, émanant des établissements d'enseignement et de recherche français ou étrangers, des laboratoires publics ou privés. 


\title{
Genetic Algorithm Coupled with a Deterministic Method for Optimization in Electromagnetics
}

\author{
J. A. Vasconcelos $\quad$ R. R. Saldanha \\ Electrical Eng. Department - UFMG - Brazil \\ L. Krähenbühl A Nicolas \\ Centre de Génie Electrique de Lyon - ECL - France
}

\begin{abstract}
In this paper, a hybrid technique for global optimization based on the genetic algorithm and a deterministic method is presented. A potential advantage of the hybrid method compared to the genetic algorithm is that global optimization can be performed more efficiently. An intrinsic problem of the hybrid techniques is related to the moment of stopping the stochastic routine to launch the deterministic one. This is investigated using some natural criteria for the commutation between the two methods. The results show that it is possible to gain in efficiency and in accuracy but the criterion is usually problem dependent. Finally, to show the solution of a real problem, the hybrid algorithm is coupled to a $2 \mathrm{D}$ code based on the boundary element method to optimize a connector of $145 \mathrm{kV}$ GIS.
\end{abstract}

\section{INTRODUCTION}

Among the optimization methods, two great families stand out: the deterministic and the stochastic. The deterministic techniques seek the minimum point based on the information given by the negative of the gradient (or sub-gradient) of the objective function. Naturally, the efficiency of them depends on several factors, such as the starting point, the accuracy of the descent direction evaluation and the method used to execute the line search as well as the stopping criteria [1]. The obtained solution point is generally a point of local minimum, which may also be a global minimum if the function is unimodal. The two main disadvantages are the need of gradient evaluations and the lack of guarantee of the global minimum. The stochastic methods do not need the calculation of the gradient and are able to find the global solution. However, the number of evaluations of the objective function, necessary to find the solution, is normally higher than the number required by the deterministic methods.

The commutation condition to stop the genetic algorithm (GA) [2] to launch the deterministic one is the major problem when dealing with such hybrid techniques. The main idea is to launch the deterministic method after the region of global minimum has been found. However, when this is achieved is not known. The goal is to explore the good characteristics of both methods. That is, global convergence with a relatively small number of objective function evaluation and with accuracy in the solution.

In recent years, papers on hybrid methods have been published, but only a few suggestions on the commutation problem have been given, for the simulated annealing algorithm [3].

Manuscript received March 19,1995. This work was supported in part by CNPq and FAPEMIG - Brazil. The email address of the authors are: joao@cpdee.ufing.br, rodney@cpdee.ufimg.br, krahenb@trotek.ec-lyon.fr and alain@trotek.ec-lyon.fr.

\section{Genetic Algorithim}

Genetic algorithms have been successfully applied to optimization problems in many areas. The important work of J. Holland [2] set up the basis of the GA and D. Goldberg's book [4] was very important to make it popular. Many papers using GA to optimize problems in others areas, such that power flow and machine learning, can easily be found in the literature. Although GA was known since 1975, the first papers in the literature applying GA in electromagnetics were only published in $1994[5,6]$.

The GA's act on a set of possible solutions, usually refereed to as a population of individuals, in such a way that the individuals of a new generation carry out their missions better than their ancestors.

In a genetic code, some operators similar to those of natural genetics are used. The main operators which affect the make up of a "chromosome" (one possible solution of the problem normally codified in binary code) are crossover, mutation and inversion $[2,4]$. Also, in a similar way to natural evolution, the individuals with best performance have a greater probability to survive. This operation is known in a GA as reproduction.

During the process of reproduction, the probability of survival is strictly linked to the performance of each individual. Those that carry out their missions better than others have a greater probability of transmitting their genetic material to the next generation. The performance of an individual is evaluated by a function that is called fitness function.

Crossover is the most important operation to generate new points in the feasible domain in the search for the global minimum. This operation is made between pairs of parents, which are randomly chosen. The exchange of genetic material is carried out and the two individuals generated are then carriers of part of genetic characteristics of their parents. New points in the feasible space are generated.

Mutation is the most important operation to introduce new genetic characteristics in the population. It occurs on an individual at a time with a probability $\mathrm{pm}$.

Naturally, if these operations are to be used with a view to optimization, the possible solutions of the problem (sets of design variables) should be coded as a finite-length string, such as a chromosome. A string of binary characters would be an example.

Codifying the Design Variables - Using binary representation, the set of design variables are then represented by a string of characters, on which natural operations are carried out [4]. 
As an example, the parameter radius of a curve $r$, which is bounded below and upper by $r_{\min }$ and $r_{\max }$, may be represented by a string of characters $A$ with a length $l=5$. Obviously, $A_{S}=11111$ and $A_{i}=00000$ match $r_{\max }$ and $r_{\min }$, respectively. The corresponding value of $r$, in the binary code, is mapped between As and Ai.

The string length is chosen according to the precision required for the parameter representation. In the example above, if the radius $r$ is represented by $A=01010$ and the limits $r_{\min }$ and $r_{\max }$ are 1 and $110 \mathrm{~cm}$, the value of $r$ is $1+(109 / 31)^{*} 10$ $\mathrm{cm}$ because $\mathrm{A}, \mathrm{A}_{\mathrm{i}}$ and $\mathrm{A}_{\mathrm{S}}$ are respectively in base 10 equal to 10,0 and 31 . The resolution for this representation is the ratio $\left(r_{\max }-r_{\min }\right) /\left(A_{S}-A_{j}\right)$, which gives $109 / 31$.

When there are several design variables, they can be represented by a single string of binary digits [4]: the first variable takes the first lo positions, the second takes the next lo positions, etc. The picture below illustrates a string with $n$ optimization variables each one of them represented in binary code as $l o=7$. The advantage is that each string represents a possible solution to the problem, based on which all the operations can be done. However, each variable may be left independent and the operations carried out separately. Thus, the first parameter of the first string can be crossed with the first parameter of the last, at the same time as the second variable of the first string can be crossed with the second parameter of the third. This representation may have some advantages because the possibility of testing new solutions is greatly increased. Our GA code was implemented using this last option [7]. This representation has the inconvenience of needing a vector to represent a possible solution.

$$
A=\underset{\text { par } 1}{10} \underset{\text { par } 2}{10101100100101 \ldots 101001}
$$

\section{GenentC Algorrthm Implementation}

The simplified organogram of a genetic algorithm, for the optimization of a constrained non-linear problem, was changed to permit the commutation between the GA and a deterministic method [7]. Also, in this new implementation, the $p_{m}$ and $p_{c}$ probabilities are dynamically changed based on the rate $\mathrm{V}_{\text {aver }} / \mathrm{V}_{\max }$, where $\mathrm{V}_{\text {aver }}$ is the fitness function mean value and $V_{\max }$ is the best fitness function value. When this rate is close to one, there is not much genetic diversity in the population. So $p_{m}$ is augmented and $p_{c}$ is lowered and when this rate is close to zero, this means that there is a lot of genetic variety in the population. In this case, $p_{m}$ is lowered and $p_{c}$ is augmented. Although these last changes have been tested only in the solution of some analytical functions, the GA code has given better results, presented more stability and the convergence has been accelerated. A detailed study about these changes has actually been made. Finally, the inversion process was implemented in our GA, without changes in the way that the variables were coded. So, when $\mathrm{p}_{\text {ivv }}>0$, the "inversion" process generates new points in the feasible space.
PROCEDURES

\begin{tabular}{|c|c|c|}
\hline 1 & $\begin{array}{l}\text { Choose: nbpop, nbgen, pc, pm, pinv, } \varepsilon, \\
\text { Do: }=0 \\
\text { Choose: } x_{i}^{1} \quad \mathrm{i}=1, \text { nbpop; }\end{array}$ & (initial population) \\
\hline 2 & Do: $t=1+1$ & (generations counter) \\
\hline 3 & Calculate $\mathrm{ff}_{;}\left(\mathrm{x}^{\mathrm{t}}\right) \quad \mathrm{i}=1, \mathrm{nbpop}$ & (fitness function calculation) \\
\hline 4 & $\begin{array}{l}\text { Select } f_{m}\left(x^{t}\right) \geq f_{i}\left(x^{t}\right) \text { for } i=1, \text { nbpop; } \\
\text { If } t>t_{0} \text { and the commutation criterion } \\
\text { is satisfied or if } t>\text { nbgen then stop GA } \\
\text { and start ALM. }\end{array}$ & $\begin{array}{l}\text { (selection of the individual } \\
\text { with the best performance) } \\
\text { (the best solution found with } \\
\text { GA is passed to the } \\
\text { deterministic method) }\end{array}$ \\
\hline 5 & $\begin{array}{r}\text { If } \mathrm{t}>1 \text { : Select } \mathrm{ff}_{r}\left(\mathrm{x}^{\mathrm{t}}\right) \leq \mathrm{ff}_{f}\left(\mathrm{x}^{\mathrm{t}}\right) \text { for } \mathrm{i}=1, \mathrm{nb} \\
\text { (Selection of indi } \\
\text { Replace } \mathrm{ff}_{r}\left(\mathrm{x}^{\mathrm{t}}\right) \text { by } \mathrm{ff}_{m}\left(\mathrm{x}^{\mathrm{t}-1}\right) \text {. } \\
\text { (Individual of worst } \\
\text { of best performanc }\end{array}$ & $\begin{array}{l}\text { ual with worse performance) } \\
\text { formance substituted by that } \\
\text { om the previous generation) }\end{array}$ \\
\hline 6 & Reproduction & (Selection of survivors) \\
\hline 7 & (Operation betw & $\begin{array}{l}\text { n pairs of individuals chosen } \\
\text { adomly with a probability pc) }\end{array}$ \\
\hline 8 & Random operatio & $\begin{array}{r}\text { on an individual chosen with a } \\
\text { probability pm) }\end{array}$ \\
\hline 9 & Random operation & $\begin{array}{r}n \text { an individual chosen with a } \\
\text { probability pinv) }\end{array}$ \\
\hline
\end{tabular}

\section{FITNESS FunCTION DeFINITION}

Any code for electromagnetic field calculation can be coupled to a genetic algorithm through an objective function. As long as the GA is established naturally in terms of maximization with a non-negative function in the feasible domain of the problem, it is necessary to map the original objective function in another way to adapt it to the $G A$. Non-linear optimization problems in electromagnetism can be expressed as:

$$
\begin{array}{ll}
\min & f(\mathbf{x}) \\
\text { subjected to } & g_{i}(\mathbf{x}) \leq 0 \quad i=1, m
\end{array}
$$

where $f(x)$ is the objective function, $g_{i}(x)$ is the $\mathrm{i}$-th constraint function and $x=\left(x_{1}, x_{2}, \ldots, x_{n}\right)$ is the vector of design variables.

Using a transformation as in the penalty methods, the original problem (Eq. (1)) can be rewritten as a problem of unconstrained minimization.

$$
\min h(\mathbf{x})=f(\mathbf{x})+r \sum_{i=1}^{m_{2}}\left[g_{i}(\mathbf{x})\right]_{+}
$$

In the equation (2), $\mathrm{r}$ is a penalty parameter, the term $\left[\mathrm{g}_{i}(\mathbf{x})\right]_{+}$ represents only the violated constraint functions and $h(x)$ is a pseudo-objective or a cost function.

The problem above can be transformed into an adequate form to the genetic algorithm, i.e., into a form of maximization $[1,5]$ :

$$
\max f f(\mathbf{x})=\frac{1}{[h(\mathbf{x})+\varepsilon]}
$$

where $\varepsilon$ is simply a constant that should be chosen in such a way that in global minimum point $h\left(x^{*}\right)+\varepsilon \cong \rho$, where $\rho$ is a constant slightly higher than zero $\left(10^{-6}\right)$ and $f(x)$ is the fitness function. The analysis of some results using this function has shown that the GA convergence is accelerated. This can be 
explained with regards to the reproduction process: when a point, say $x_{1}$, in the feasible space, is generated in such a way that $h\left(\mathrm{x}_{1}\right)+\varepsilon$ is close to zero, it will have a great probability to be reproduced many times, because $f\left(x_{1}\right)$ is the measure of the individual performance associated with this point.

\section{Commutation Crtterta}

The major difficulty when dealing with hybrid methods for global optimization problems is to find the moment to stop the stochastic method to launch the deterministic one. The main idea is to start the deterministic method when the region of global minimum has been found. However, it is not known when this is achieved. So, it is impossible to guarantee that the solution is the global one. The results presented in next section illustrates this fact.

The following criteria for the commutation between GA and a deterministic method can be naturally defined:

A. Number of generations. It is the simplest case. The GA is stopped when the specified number of generations is attained and the best result is passed to the deterministic method. The major drawback of this criterion is that not all cost functions present the same behavior.

B. Difference between the cost function mean values at a set of generations. The major inconvenience of this criterion is that the evolution of the mean value curve presents great variations from one generation to another.

C. Difference between the best values of the cost function at a set of generations. This procedure is less sensitive to the type of the problem and the condition can be established as:

$\left|t_{o} h_{m}^{t}-\sum_{j=1}^{t_{o}} h_{m}^{t-j}\right| \frac{1}{\left(\varepsilon+h_{m}^{t}\right)} \leq \varepsilon_{o}$

where $t$ is the actual generation and $t_{0}$ is an integer number, which is used to indicates how many generations before $t$ will be considered. Moreover, $\mathrm{h}_{\mathrm{m}}{ }^{\mathrm{t}}$ is the best cost function value at generation $t$ and $\varepsilon_{0}$ is a small positive number.

D. Difference between the best different values of the cost function in the same generation. This procedure is also less sensitive to the type of the problem. It can be written as:

$$
\left|t_{o} h_{m}^{t}-\sum_{j=1}^{t_{o}} h_{m-j}^{t}\right| \frac{1}{\left(\varepsilon+h_{m}^{t}\right)} \leq \varepsilon_{o} \quad h_{m}^{t}<h_{m-1}^{t}<\cdots<h_{m-t_{o}}^{t}
$$

where $h_{m-j}{ }^{t}$ is the $j$-th best value after $h_{m}{ }^{t}$ and $t_{0}$ is the number of the individuals with best performances taken in the same generation for comparison.

\section{Anal ytical Results}

The problem of commutation was analyzed using the GA coupled with the augmented Lagrangian method (ALM) [1]. For this study, the problem of minimizing the Rastrigin function, defined in the interval $(0,10)$, was taken as a test problem [7]. This problem presents $10^{\mathrm{n}}$ local minima (Eq. 6).

$$
\begin{aligned}
& \min f(\mathbf{x})=10 n+\sum_{i=1}^{n}\left[\left(x_{i}-x_{o i}\right)^{2}-10 \cos 2 \pi\left(x_{i}-x_{o i}\right)\right] \\
& \mathbf{x}=\left(x_{1}, x_{2}, \ldots, x_{n}\right)^{T} \quad 0 \leq x_{i} \leq 10 \quad x_{o i} \in(0,10)
\end{aligned}
$$

The global solution $x^{*}$ is evidently $x^{*}=\left(x_{01}, x_{02}, \ldots, x_{0 n}\right)^{T}$ with the objective function having a zero value. The vector $\mathbf{x}_{0}$ permits us to choose global solutions that can be more or less difficult to the GA to find.

The Table I gives the results only for the commutation criteria A and C, although the criteria B and D have also been tested. In these tests, the criterion $B$ has not given good results because the mean value curve presents great variations [5] and the condition $\mathrm{D}$ has given similar results to the condition $\mathrm{C}$. The condition $C^{\prime}$ in Table $I$ is the same criterion as $C$, except that when the condition $\left|h_{m}{ }^{t}-h_{m}{ }^{t-1}\right|<\varepsilon_{o}$ is satisfied. In this case, $h_{m}{ }^{t-1}$ is replaced by another better value that is different from $h_{m}{ }^{t}$. The global solution was chosen as $\mathbf{x}_{0} \in(3.22,5.22,6.22,7.22,8.22)^{\mathrm{T}}$ according to $n$. If $n=2, x_{0}=(3.22,5.22)^{T}$. It can be seen that not all tested cases converged to the global solution.

\begin{tabular}{|c|c|c|c|c|c|c|c|c|}
\hline \multirow{2}{*}{$\begin{array}{l}\text { Commutation Criteria } \\
\text { Number of Variables }\end{array}$} & \multicolumn{4}{|c|}{$\mathrm{C}\left(\mathrm{to}_{\mathrm{O}}=3\right)$} & \multicolumn{4}{|c|}{$C^{\prime}\left(t_{0}=2\right)$} \\
\hline & 2 & 3 & 4 & 5 & 2 & 3 & 4 & 5 \\
\hline Convergence & yes & yes & yes & no & yes & yes & yes & yes \\
\hline GA-Final Result & 1.76 & 0.08 & 0.6 & 11.92 & 1.76 & 0.89 & 0.60 & 0.72 \\
\hline ALM- Final Result & 0.0 & 0.0 & 0.0 & 9.95 & 0.0 & 0.0 & 0.0 & 0.0 \\
\hline GA-Function evaluations & 180 & 480 & 500 & 420 & 200 & 340 & 560 & 1150 \\
\hline ALM-Function evaluations & 18 & 19 & 17 & 21 & 18 & 19 & 17 & 20 \\
\hline
\end{tabular}

TABLE I

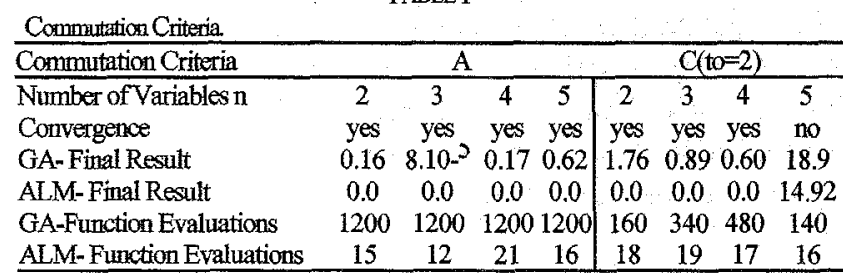

\section{Shape Optimization of a hV CONnector}

A part of a three phase $145 \mathrm{kV}$ GIS was used as a real problem to test the GA-ALM. The field computation code used is based on the boundary element method [8]. Fig. 1 shows the initial profile of a part of this connector to be optimized and Table II gives the used variable bounds. It is described using eight parameters [7].

The optimization problem was formulated as:

$$
\begin{aligned}
\min & f(\mathbf{x})=\left(E_{\max }^{2}-E_{o}^{2}\right)^{2} \\
x_{\min }^{i} & \leq x^{i} \leq x_{\max }^{i} \quad i=1,8
\end{aligned}
$$

where $\mathbf{x}$ is the vector of design parameters, $E_{0}$ is the specified electric field and $E_{\max }$ is the maximum value of this field on the profile. $\mathrm{E}_{\mathrm{o}}$ is chosen equal to $26 \mathrm{~V} / \mathrm{m}$. 


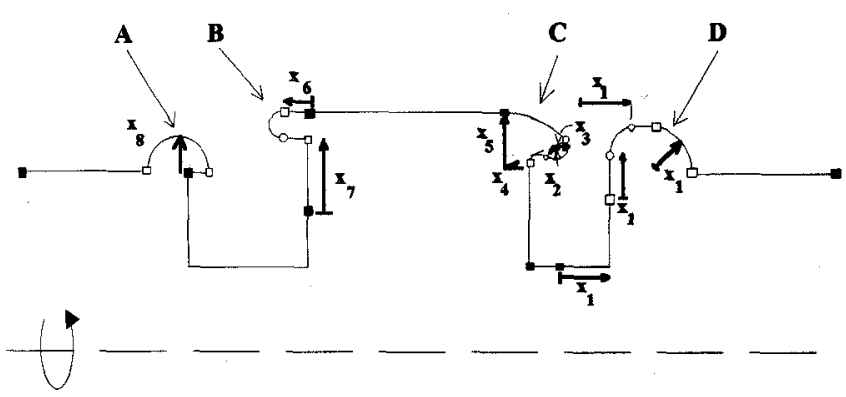

Fig. 1. Initial profile and the used parameters [7].

The genetic parameters used for this example were:

- Maximum number of generations: 20

- Number of individuals in the population: 10

- Crossover probability:

- Mutation probability:

0.025

- Inversion probability:

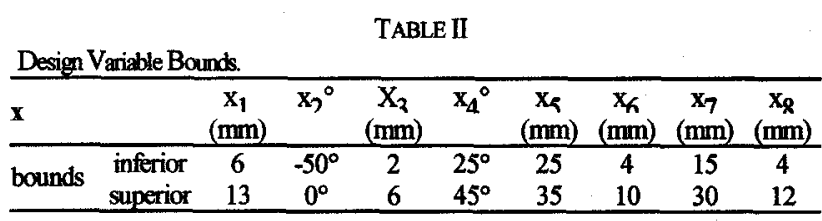

The criterion $C$ was used in this computation as shown in Eq. (8). The parameters $t_{0}, \varepsilon$ and $\varepsilon_{0}$ were respectively replaced by 2,1 and 0.001 . Also, $h(x)$ was replaced by $f(x)$ since (7) is a minimization problem without constraints. The $\varepsilon$ value was made equal to 1 , because the objective function value at the minimum point usually is not known, in such a way as to satisfy $f\left(x^{*}\right)+\varepsilon \cong \rho$.

$$
\left|2 f(\mathbf{x})_{m}^{t}-f(\mathbf{x})_{m}^{t-1}-f(\mathbf{x})_{m}^{t-2}\right| \frac{1}{\left(1+f(\mathbf{x})_{m}^{t}\right)}<0.001
$$

In Eq. (8), $f(\mathbf{x})_{m}^{t}$ is the best objective function value result obtained in the generation $\mathrm{t}$.

The results obtained are shown in Table III and Fig. 2 [7]. Table III gives the initial and the final values of the design variables after the execution of ALM and the hybrid GA-ALM. The results show that there is more than one minimum.

It is interesting to see that the four regions (A,B,C and D) shown in Fig. 1 have the largest values of the electrical field. Also, the maximum values of this field in the initial geometry are respectively $36.74,49.00,29.84$ and $36.02 \mathrm{~V} / \mathrm{m}$. In this problem, 28 field evaluations were made by the GA, which passed the best geometry found to ALM, that made 16 more evaluations. Note that the optimum point was not found by GA. The results show only that the commutation criterion was satisfied after three generations and the best result obtained with GA was passed to ALM. The $E_{\max }$ obtained with the optimization, using the hybrid GA-ALM, is $36.44 \mathrm{~V} / \mathrm{m}$ - (region B) for GA and 35.34 - (region B) for ALM.

TABLE III

Max. Normal Elect. Field Values on the HV Comector for a Diff of Pot of $1 \mathrm{~V}$

\begin{tabular}{lcccc}
\hline & Initial & ALM & \multicolumn{2}{c}{ GA-ALM } \\
\hline $\mathrm{E}_{\text {max }}(\mathrm{V} / \mathrm{m})$ (Point - Fig. 1) & $49.00(\mathrm{~B})$ & $35.40(\mathrm{~A})$ & $36.44(\mathrm{~B})$ & $35.34(\mathrm{~B})$ \\
NBCAL ( 13s/calcul) & & 14 & 28 & 16 \\
$\mathrm{x}_{1}(\mathrm{~mm})$ & 10 & 7.76 & 7.26 & 7.28 \\
$\mathrm{x}^{\circ}$ & $-30^{\circ}$ & $-30^{\circ}$ & $-13.63^{\circ}$ & $-13.64^{\circ}$ \\
$\mathrm{x}_{2}(\mathrm{~mm})$ & 4 & 4 & 5.25 & 5.25 \\
$\mathrm{x}_{4}^{\circ}$ & $35^{\circ}$ & $35^{\circ}$ & $34.13^{\circ}$ & $34.14^{\circ}$ \\
$\mathrm{x}_{5}(\mathrm{~mm})$ & 30 & 30.14 & 33.17 & 33.17 \\
$\mathrm{x}_{6}(\mathrm{~mm})$ & 6 & 10.00 & 8.36 & 10.00 \\
$\mathrm{x}_{7}(\mathrm{~mm})$ & 28 & 16.55 & 17.33 & 15.81 \\
$\mathrm{x}_{8}(\mathrm{~mm})$ & 7.5 & 10.72 & 9.80 & 10.60 \\
\hline
\end{tabular}

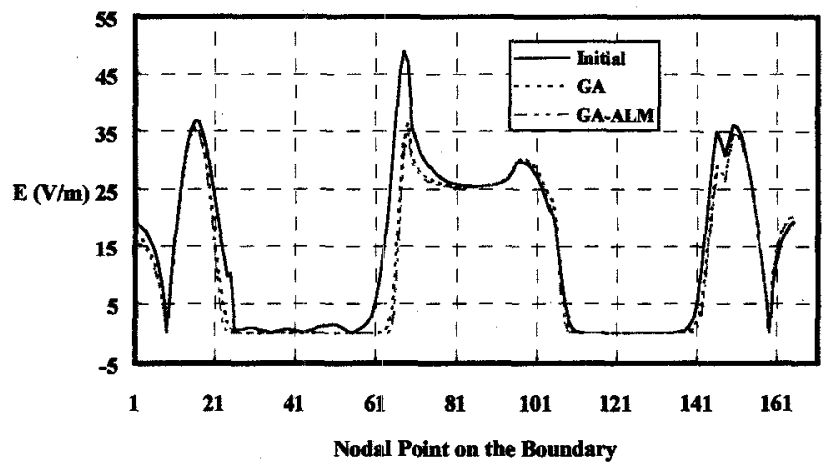

Fig. 2. E-field (initial geometry, geometry obtained with GA and passed to ALM and the final result obtained with GA-ALM).

\section{Conclusion}

A hybrid technique for global optimization problems was presented in this paper. The major difficulty to stop the GA and launch the ALM was studied using some natural commutation conditions. Although the analytical results presented show that it is possible to increase the numerical efficiency (see cases A, C and $C^{\prime}$ ), the global solution can not be guaranteed. The condition A is the simplest one. However, cost functions can present different behaviors, requiring additional tests to find the number of generations necessary to achieve the global region. The condition $C$ is less sensitive to the problem type and can be adjusted changing the value of $t_{0}$. The optimized shape of the HV connector above illustrates the application of the hybrid method to the solution of a real problem.

\section{REFERENCES}

[1]J. A. Vasconcelos, L. Krahenbuhl, L. Nicolas and A. Nicolas, "Design optimization in electrostatic field analysis using the BEM and the augmented Lagrangian method", IEEE Trans. on Magnetics, vol. 30, pp. 3443-3446, September 1994.

[2]J. H. Holland, "Adaptation in natural and artificial systems", MIT Press, 1992

[3]G. Drago, A. Manella, M. Nervi, M. Repetto and G. Secondo, "A combined strategy for optimization in non linear magnetic problems using simulated annealing and search techniques", IEEE Trans. on Magnetics, vol. 28, pp. 1541-1544, 1992.

[4]D. E. Goldberg, "Genetic Algorithms in Search, Optimization \& Machine Learming", Addison-Westey, 1989

[5]J.A. Vasconcelos, L. Krähenbuhl, L. Nicolas and A Nicolas, "Design optimisation using the BEM coupled with Genetic Algorithm", Proc. of the 2nd IEE Int. Conf. on Computation in Electromagnetics, pp. 60-63, April 1994.

[6]G.F. Uller, O.A. Mohammed and C.S. Koh, "Utilizing genetic algorithms for the optimal design of electromagnetic devices", IEEE Trans. on Magnetics, vol. 30, pp. 4296-4298, November 1994.

[7]J. A. Vasconcelos, "Optimisation de forme des structures électromagnétiques", Ph.D. Thesis, Ecole Centrale de Lyon, Ecully, France, July 1994.

[8]J. A. Vasconcelos, L. Nicolas, F. Buret and A. Nicolas, "Shape optimization of an HV connector in a GIS", Electric and Magnetic Fields from Numerical Models to Industrial Applications, Plenum Press, pp. 277-280, 1995. 